\title{
Task Mapping Algorithm for Heterogeneous Computing System Allowing High Throughput and Load Balancing ${ }^{1}$
}

\author{
Sung Chune Choi and Hee Yong Youn \\ School of Information and Communications Engineering, \\ Sungkyunkwan University, \\ 440-746, Suwon, Korea +82-31-290-7952 \\ \{choisc, youn\}@ece.skku.ac.kr
}

\begin{abstract}
The applicability and strength of heterogeneous computing systems are derived from their ability to match computing nodes to appropriate tasks since a suite of different machines are interconnected. A good mapping algorithm offers minimal expected completion time and machine idle time. In this paper we propose a new task scheduling algorithm allowing higher performance than the existing algorithms such as the Min-min, Max-min, and Sufferage algorithm. It is achieved by task swapping approach based on the expected completion time and ready time of each machine. Extensive computer simulation validates the proposed algorithm along with the earlier ones.
\end{abstract}

Keywords: Heterogeneous computing, load balancing, task mapping, throughput, scheduling.

\section{Introduction}

In heterogeneous computing (HC) environment, a suite of different machines are interconnected to provide a variety of computational capabilities and maximize their combined performance to execute tasks having diverse requirements. There exist a number of different types of HC systems. This paper focuses on mixed-machine HC systems, where a number of high-performance heterogeneous machines are interconnected through high-speed links [1].

In $\mathrm{HC}$ system the application is decomposed into tasks, where each task is computationally homogeneous. The applicability and strength of $\mathrm{HC}$ systems are derived from their ability to match the computing resources to appropriate tasks. Here each task is assigned to one of the machines which is best suited for its execution to minimize the execution time. Therefore, an efficient mapping scheme allocating the application tasks to the machines is needed.

The general problem of mapping tasks to the machines is a well known NPcomplete problem and several mapping algorithms have been proposed to approxi-

\footnotetext{
${ }^{1}$ This research was supported by the Ubiquitous Autonomic Computing and Network Project, 21st Century Frontier R\&D Program in Korea and the Brain Korea 21 Project in 2004. Corresponding author: Hee Yong Youn.
} 
mate its optimal solution in the literature. The representative batch mode mapping algorithms are Min-min, Max-min, and Sufferage algorithm [2,3,4]. A good mapping algorithm compromises between matching for smallest expected completion time and load balancing to minimize the machine idle time. Since the previous algorithms have some limitations, we propose a new scheduling algorithm solving them. It is achieved by task swapping approach based on the expected completion time and ready time of each machine. Computer simulation reveals that the proposed algorithm consistently outperforms the earlier algorithms for various degree of task and machine heterogeneity.

\section{Related Work}

At first, we define some metrics used throughout the paper, which are the expected execution time (EET), ready time (RT), expected completion time (ECT), and makespan. The $\mathrm{EET}_{i j}$ is the estimated execution time for task $i\left(t_{i}\right)$ on machine $j\left(m_{j}\right)$ if $\mathrm{m}_{j}$ has no load when $t_{i}$ is assigned. If an HC system of $m$ machines has $t$ tasks, we can obtain a $t \times m$ EET matrix. The $\mathrm{RT}_{j}$ is the time that $m_{j}$ becomes ready after completing the execution of the tasks that are currently assigned to it. The $\mathrm{ETC}_{i j}$ is the time at which $m_{j}$ completes $t_{i}$ after finishing any previously assigned tasks. From the definitions above, it is easy to get $\mathrm{ECT}_{i j}=\mathrm{RT}_{i}+\mathrm{EET}_{i j}$. The makespan for a complete schedule is then defined as the time duration from the start to the time the entire tasks are completed. Makespan is a measure of the throughput of an algorithm. In other words, the ready time of a machine after tasks are assigned will be smaller if the makespan is decreased.

The Min-min algorithm computes each task's Minimum Completion Time (MCT) over the available hosts and the task with the minimum MCT is assigned to the best host. The motivation behind the Min-min algorithm is that assigning tasks to the hosts completing them fastest will lead to overally reduced makespan. The Max-min algorithm is similar to the Min-min algorithm except that task with the maximum earliest completion time is assigned. The Max-min algorithm might outperform the Min-min algorithm when there exist more short tasks than long tasks. The Sufferage algorithm assigns a machine to a task that would 'suffer' most in term of expected completion time if that particular machine is not assigned to it.

\section{The Proposed Scheduling Algorithm}

The proposed new scheduling algorithm shown in Figure 1 is divided into two parts. The initialization step of Line (1) to (4) is similar to the ones in the Min-min and Max-min algorithm. It differs from the Min-min algorithm in that a task is mapped onto a machine as soon as it arrives at the scheduler like the MCT (minimum completion time) algorithm. Each task is examined to determine the machine providing earliest completion time, and then the pre-allocation table and the temporary RT table are updated to reassign the tasks. The MCT algorithm is fast and simple, but it may not assign a task to the best matched machine since it does not consider subsequently arriving tasks those better match the machine. To remedy this sort of miss-matching, 
each task is reassigned by calculating the expected completion time and the ready time of each machine in Line (5) to (13). In each iteration of the for loop, a task is selected arbitrarily, and the task having earlier completion time than this one is found if it exists. If such task exists, the two tasks are swapped if at least one of the following two conditions is satisfied; i) the ECTs of the two machines are decreased, ii) even though the ECT of one machine is increased, the maximum ready time is decreased. The main objective of the proposed approach is for better matching and load balancing at the same time. When all the iterations of the inner for loop are completed, the temporary RT table of each machine is updated.

/* a task is mapped onto a machine as soon as it arrives at the scheduler */

(1) for all $\mathrm{m}_{j}$ (in a fixed arbitrary order)

(2) calculate the ECT according to EET and RT, and then update

(3) find the machine $\mathrm{m}_{j}$ with the minimum earliest completion time

(4) update the temporary RT table and update the pre-allocation table

(5) for each $\mathrm{t}_{k}$ that assigned to each machine (in a fixed arbitrary order)

(6) for each task $\mathrm{t}_{i}$ for $\mathrm{m}_{j}$ (in a fixed arbitrary order)

(7) calculate the diff_value of $\mathrm{t}_{i}$ and $\mathrm{t}_{k}$ to $\mathrm{m}_{j}$ and $\mathrm{m}_{l}$

(8) if (Temporary $\left.\mathrm{RT}_{l}+\mathrm{EET}_{k l}\right)<($ Maximum ready time in temporary $\mathrm{RT}$ table) $\& \&$ diff_value of task $\mathrm{t}_{i}$ assigned to $\mathrm{m}_{j} \geq$ the diff_value of $\mathrm{m}_{l}$

(9) swap task between $\mathrm{m}_{j}$ and $\mathrm{m}_{l}$

(10) for every machine $\mathrm{m}_{j}$

(11) if(Temporary $\left.\mathrm{RT}_{j}+\mathrm{EET}_{k j}\right)<($ Maximum ready time in temporary $\mathrm{RT}$ table)

(12) deallocate $t_{k}$ and allocate $t_{k}$ to $\mathrm{m}_{j}$

(13) update the temporary RT table

(14) update the ready time table based on the tasks that were assigned to the machines

(15) update the expected completion time table

Fig. 1. The proposed scheduling algorithm

\section{Performance Evaluation}

Table 1 shows the improvement of the EET of the Max-min, Min-min, and Sufferage algorithm using the proposed algorithm for eight machine system. Here,

Table 1. Improvement of the EETs with the proposed algorithm for eight machine system

\begin{tabular}{|c|c|c|c|}
\hline Task/ Machine heterogeneity & Max-min & Min-min & Sufferage \\
\hline Low/ Low & $30.1 \%$ & $3.5 \%$ & $3.4 \%$ \\
\hline Low/ High & $27.6 \%$ & $3.7 \%$ & $5.3 \%$ \\
\hline High/ Low & $26.7 \%$ & $1.3 \%$ & $0.5 \%$ \\
\hline High/ High & $29 \%$ & $3.7 \%$ & $2.6 \%$ \\
\hline
\end{tabular}


consistent EET table was obtained from the inconsistent EET table by sorting the execution times of the tasks on all machines. Note that the proposed algorithm outperforms the existing algorithms for various degree of task and machine heterogeneity.

\section{Conclusion}

The mapping algorithms in distributed systems aim at different measure such as makespan, load balancing, and throughput. A good mapping algorithm needs to compromise the conflicting measures. In this paper we have proposed a new task scheduling algorithm which is better than the existing algorithms in terms of throughput and load balancing. It is achieved by task swapping approach based on the expected completion time and ready time of each machine. In inconsistent heterogeneity mode, the makespan of the proposed algorithm is lower than the existing algorithms because it swaps the tasks among the machines for archiving good load balancing. Also, the proposed algorithm has lower time complexity than others.

As a future work we will develop a more optimized algorithm that considers the quality of service, and carry out comprehensive performance evaluation. We will also implement the newly proposed task scheduling algorithm in an actual heterogeneous environment for testing and refinement.

\section{References}

1. H. J. Siegel, J. K. Antonio, R. C. Metzger, M. Tan, and Y. A. Li, "Heterogeneous computing." In A. Y. Zomaya (ed.), Parallel and Distributed Computing Handbook, New York, NY: McGraw-Hill, 1996, 725-761.

2. Maheswaran, M. Ali, S. Siegal, H.J. Hensgen, D. Freund, R.F., "Dynamic matching and scheduling of a class of independent tasks onto heterogeneous computting systems," HCW'99, 1999, 30-44.

3. O.H. Ibarra and C. E. Kim, "Heuristic algorithms for scheduling independent tasks on nonidentical processors," Journal of the ACM, 24(2), April 1977, 280-289.

4. R. F. Freund, M. Gherrity, S. Ambrosius, M. Campbell, M. Halderman, D. Hensgen, E. Keith, T. Kidd, M. Kussow, J. D. Lima, F. Mirabile, L. Moore, B. Rust, and H. J. Siegel, "Scheduling resources in multiuser, heterogeneous, computing environments with SmartNet," HCW'98, 1998, 184-199.

5. Ali, S. Siegel, H.J. Maheswaran, M. Hensgen, D. "Task execution time modeling for heterogeneous computing systems," HCW'2000, May 2000, 185 - 199

6. M.Y. Wu and W. Shu, "A High-Performance Mapping Algorithm for Heterogeneous Computing Systems," International Parallel and Distributed Processing Symposium (IPDPS), April 2001.

7. Arnaud Giersch, Yves Robert, Frédéric Vivien, "Scheduling Tasks Sharing Files on Heterogeneous Master-Slave Platforms", 12th Euromicro Conference on Parallel, Distributed and Network-Based Processing (PDP'04), February 2004, 11 - 13. 